\title{
On the Spectra of Commuting and Non Commuting Graph on Dihedral Group
}

\author{
Abdussakir ${ }^{1}$, Rivatul Ridho Elvierayani², Muflihatun Nafisah ${ }^{3}$ \\ 1,2,3) Mathematics Department, Universitas Islam Negeri Maulana Malik Ibrahim Malang \\ Email: sakir@mat.uin-malang.ac.id
}

\begin{abstract}
Study about spectra of graph has became interesting work as well as study about commuting and non commuting graph of a group or a ring. In this paper, we investigate adjacency spectrum, Laplacian spectrum, signless Laplacian spectrum, and detour spectrum of commuting and non commuting graph of dihedral group $D_{2 n}$.
\end{abstract}

Keywords: graph, spectrum, commuting graph, non commuting graph, dihedral group.

\section{INTRODUCTION}

Let $G$ be a graph of order $n(n \geq 1)$ and let its vertex set is $V(G)=\left\{v_{1}, v_{2}, \ldots, v_{n}\right\}$. The adjacency matrix $A(G)=\left[a_{i j}\right]$ of $G$ is $n \times n$ matrix where $a_{i j}=1$ if $v_{i} v_{j} \in E(G)$ and $a_{i j}=0$ if $v_{i} v_{j} \notin$ $E(G))$ [1]. So, the adjacency matrix of a graph is real and symmetric that contains 0 in all of main diagonal entries [2]. The degree matrix $D(G)=\left[d_{i j}\right]$ of $G$ is a diagonal matrix where $d_{i i}=$ $\operatorname{deg}_{G}\left(v_{i}\right)$ and $d_{i j}=0$, for $i \neq j$. The Laplacian matrix $L(G)$ of $G$ is defined by $L(G)=D(G)-A(G)$ [3] and the signless Laplacian matrix $Q(G)$ of $G$ is defined by $Q(G)=D(G)+A(G)$ [4]. The detour matrix $D D(G)=\left[e_{i j}\right]$ of $G$ is $n \times n$ matrix where $e_{i j}$ is equal to the length of the longest path between $v_{i}$ and $v_{j}[5]$.

Let $\lambda_{0}, \lambda_{1}, \ldots, \lambda_{k-1}$ where $\lambda_{0}>\lambda_{1}>\ldots>\lambda_{k-1}$ are distinct eigenvalues from a matrix of a graph, and let $m\left(\lambda_{0}\right), m\left(\lambda_{1}\right), \ldots, m\left(\lambda_{k-1}\right)$ are multiplicities of $\lambda_{i}$, for $i=0,1,2, \ldots, k-1$. The multiplicity of $\lambda_{i}$ as roots of characteristic equation is equal to the dimension of the space of eigenvectors corresponding to $\lambda_{i}$ [6]. Matrix of order $2 \times k$ that contains $\lambda_{0}, \lambda_{1}, \ldots, \lambda_{k-1}$ for the first row and $m\left(\lambda_{0}\right), m\left(\lambda_{1}\right), \ldots, m\left(\lambda_{k-1}\right)$ for the second row is called spectrum of graph $G$ and denoted by $\operatorname{Spec}(G)$ [6]. According to Yin [7], we can write spectrum of graph $G$ by

$$
\operatorname{Spec}(G)=\left[\begin{array}{cccc}
\lambda_{0} & \lambda_{1} & \cdots & \lambda_{k-1} \\
m\left(\lambda_{0}\right) & m\left(\lambda_{1}\right) & \cdots & m\left(\lambda_{k-1}\right)
\end{array}\right] .
$$

Spectrum that obtained from the adjacency matrix $A(G)$, the Laplacian matrix $L(G)$, the signless Laplacian matrix $Q(G)$, and the detour matrix $D D(G)$ are called the adjacency spectrum denoted by $\operatorname{Spec}_{A}(G)$, the Laplace spectrum denoted by $\operatorname{Spec}_{L}(G)$, the signless Laplacian spectrum denoted by $\operatorname{Spec}_{Q}(G)$, and the detour spectrum denoted by $\operatorname{Spec}_{D D}(G)$ respectively.

Let $G$ be a non Abelian group with center $Z(G)$. The commuting graph $C(G, X)$, where $X$ is a subset of $G$, is the graph with vertex set $X$ and distinct vertices being joined by an edge whenever $x y=y x$ in $G$ [8]. In the case $X=G$, we denote the commuting graph of $G$ by $C(G)$ 
instead of $C(G, X)$. The non commuting graph $\Gamma_{G}$ of $G$, is the graph whose vertices are the non central elements $G Z(G)$ and whose edges join those vertices $x, y \in G Z(G)$ for which $x y \neq y x$ in $G$ [9]. The non commuting graph $\Gamma_{G}$ of $G$ is always connected [10].

The study of spectrum of graph was begun by work of Norman Bigg [6] in his famous book Algebraic Graph Theory. Some investigations about spectrum of graphs had been done before, for example Yin [7], Brouwer and Haemers [4], Ayyaswamy \& Balachandran [5], and Jog \& Kotambari [11]. Researches on commuting and non commuting graph of a group and ring had also been done before, for example Akbari et al. [12], Abdollahi et al. [10], Darafsheh [13], Abdollahi et al. [9], Vahidi \& Talebi [14], Rahayuningtyas et al. [15], Chelvam et al. [16], Woodcock [17], and Nawawi \& Rowley [8]. In this study, we determine the spectra of commuting and non commuting graph of dihedral group, including adjacency spectrum, Laplacian spectrum, signless Laplacian spectrum, and detour spectrum.

\section{RESULT AND DISCUSSION}

Here we present several results from our studies. For adjacency spectrum, we observe and get result just for non commuting graph of dihedral group.

\section{Lemma 1:}

Let $\Gamma_{D_{2 n}}$ be a non commuting graph of dihedral group $D_{2 n}$ where $n$ is odd positive integer and $n>3$. The characteristic polynomial for adjacency matrix $A\left(\Gamma_{D_{2 n}}\right)$ of $\Gamma_{D_{2 n}}$ is

$$
p(\lambda)=(\lambda)^{n-2}(\lambda+1)^{n-1}\left(-\lambda^{2}+(n-1) \lambda+(n(n-1))\right) .
$$

\section{Proof:}

For dihedral group $D_{2 n}=\left\{1, r, r^{2}, \ldots, r^{n-1}, s, s r, s r^{2}, \ldots, s r^{n-1}\right\}$ where $n$ is odd positive integer, $n>3$, we have $Z\left(D_{2 n}\right)=\{1\}$. So, the non commuting graph $\Gamma_{D_{2 n}}$ of $D_{2 n}$ has $\{r$, $\left.r^{2}, \ldots, r^{n-1}, s, s r, s r^{2}, \ldots, s r^{n-1}\right\}$ as its vertex set. Because $n$ is odd, we have $s r^{i} \neq r^{i} s, i=$ $1,2, \ldots, n-1$, so $s$ and $r^{i}$ are adjacent in $\Gamma_{D_{2 n}}$. And also, $s r^{i} s r^{j} \neq s r^{j} s r^{i}$, for $i=1,2, \ldots$, $n-1$ and $j=1,2, \ldots, n-1$, where $i \neq j$, so $s r^{i}$ and $s r^{j}$ are adjacent in $\Gamma_{D_{2 n}}$. Then, we have the adjacency matrix for $\Gamma_{D_{2 n}}$ as the following

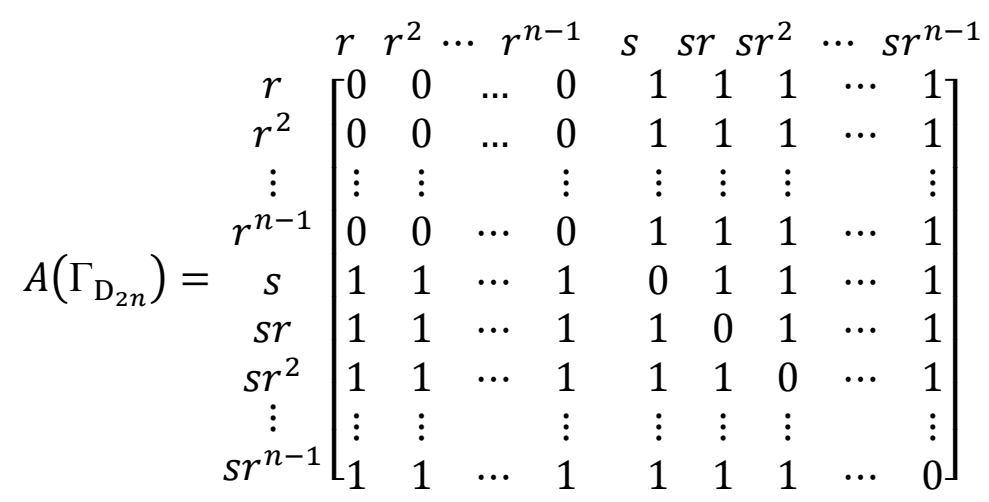

We know that the characteristic polynomial of $A\left(\Gamma_{D_{2 n}}\right)$ is $\operatorname{det}\left(A\left(\Gamma_{D_{14}}\right)-\lambda I\right)$. Using the Gaussian elimination procedure we obtain this upper triangular matrix 


\begin{tabular}{|c|c|c|c|c|c|c|c|c|c|}
\hline & 1 & & $r^{2} .$. & $r^{n-1}$ & 1 & $s r$ & $s r^{2}$ & ... & $s r^{n-1}$ \\
\hline$r$ & $\lceil 1$ & 1 & $\ldots$ & 1 & $-\lambda$ & 1 & 1 & $\cdots$ & 1 \\
\hline$r^{2}$ & 0 & $\lambda$ & $\ldots$ & $\lambda$ & $1-\lambda^{2}$ & $1+\lambda$ & $1+\lambda$ & $\cdots$ & $1+\lambda$ \\
\hline$\vdots$ & $\vdots$ & $\vdots$ & & $\vdots$ & $\vdots$ & $\vdots$ & $\vdots$ & & $\vdots$ \\
\hline$r^{n-1}$ & 0 & 0 & $\cdots$ & $\lambda$ & $(n-2)-\lambda^{2}$ & $(n-2)+\lambda$ & $(n-2)+\lambda$ & $\cdots$ & $(n-2)+\lambda$ \\
\hline$s$ & 0 & 0 & $\cdots$ & 0 & $1+\lambda$ & $-\lambda-1$ & 0 & $\cdots$ & 0 \\
\hline$s r$ & 0 & 0 & $\cdots$ & 0 & 0 & $1+\lambda$ & $-\lambda-1$ & $\cdots$ & 0 \\
\hline$s r^{2}$ & 0 & 0 & $\cdots$ & 0 & 0 & 0 & $1+\lambda$ & $\ldots$ & 0 \\
\hline$\vdots$ & $\vdots$ & $\vdots$ & & $\vdots$ & $\vdots$ & $\vdots$ & $\vdots$ & & $\vdots$ \\
\hline$s r^{n-1}$ & 0 & 0 & $\ldots$ & 0 & 0 & 0 & 0 & .. & $-\lambda^{2}+(n-1) \lambda+(n(n-1))$ \\
\hline
\end{tabular}

Because the determinant for this upper triangular matrix is the multiplication of all entries in its main diagonal, so we obtain the characteristic polynomial of $A\left(\Gamma_{\mathrm{D}_{2 n}}\right)$ as follows

$$
p(\lambda)=(\lambda)^{n-2}(\lambda+1)^{n-1}\left(-\lambda^{2}+(n-1) \lambda+(n(n-1))\right)
$$

\section{Theorem 1:}

Let $\Gamma_{D_{2 n}}$ be a non commuting graph of dihedral group $D_{2 n}$ where $n$ is odd positive integer and $n>3$. The adjacency spectrum of $\Gamma_{D_{2 n}}$ is

$$
\operatorname{Spec}_{A}\left(\Gamma_{D_{2 n}}\right)=\left[\begin{array}{cccc}
\frac{n-1}{2}+\sqrt{(n-1) n+\left(\frac{n-1}{2}\right)^{2}} & 0 & -1 & \frac{n-1}{2}-\sqrt{(n-1) n+\left(\frac{n-1}{2}\right)^{2}} \\
1 & n-2 & n-1 & 1
\end{array} .\right.
$$

\section{Proof:}

From Lemma 1, the characteristic polynomial of $A\left(\Gamma_{\mathrm{D}_{2 \mathrm{n}}}\right)$ is

$$
p(\lambda)=(\lambda)^{n-2}(\lambda+1)^{n-1}\left(-\lambda^{2}+(n-1) \lambda+(n(n-1))\right)
$$

By solving the equation $p(\lambda)=0$, we have

$$
\lambda_{1}=\frac{n-1}{2}+\sqrt{(n-1) n+\left(\frac{n-1}{2}\right)^{2}}, \lambda_{2}=0, \lambda_{3}=-1, \lambda_{4}=\frac{n-1}{2}-\sqrt{(n-1) n+\left(\frac{n-1}{2}\right)^{2}}
$$

as eigenvalues for $A\left(\Gamma_{\mathrm{D}_{2 n}}\right)$. Now, we will determine the multiplicity for each eigenvalue.

Because the multiplicity is equal to number of basis for space of eigenvectors corresponding to $\lambda_{i}, i=1,2,3$, and 4 , we substitute $\lambda_{i}$ to $A\left(\Gamma_{\mathrm{D}_{2 \mathrm{n}}}\right)-\lambda_{i} I$ and apply GaussJordan elimination to this matrix to obtain row-reduced eselon matrix and then we can see the number of zero rows of it.

For $\lambda_{1}=\frac{n-1}{2}+\sqrt{(n-1) n+\left(\frac{n-1}{2}\right)^{2}}$, by eliminating $A\left(\Gamma_{\mathrm{D}_{2 n}}\right)-\lambda_{1} I$, we obtained rowreduced eselon matrix with one zero row. So, for $\lambda_{1}=\frac{n-1}{2}+\sqrt{(n-1) n+\left(\frac{n-1}{2}\right)^{2}}$, we have its algebraic multiplicity is 1 .

For $\lambda_{2}=0$, by eliminating $A\left(\Gamma_{\mathrm{D}_{2 \mathrm{n}}}\right)-\lambda_{2} I$, we obtain row-reduced eselon matrix with $n$ -2 zero rows. So, for $\lambda_{2}=0$, we have its algebraic multiplicity is $n-2$.

For $\lambda_{3}=-1$, by eliminating $A\left(\Gamma_{\mathrm{D}_{2 n}}\right)-\lambda_{3} I$, we obtain row-reduced eselon matrix with $n-1$ zero rows. So, for $\lambda_{2}=-1$, we have its algebraic multiplicity is $n-1$. 
For $\lambda_{4}=\frac{n-1}{2}-\sqrt{(n-1) n+\left(\frac{n-1}{2}\right)^{2}}$, by eliminating $A\left(\Gamma_{\mathrm{D}_{2 \mathrm{n}}}\right)-\lambda_{4} I$, we obtain rowreduced eselon matrix with one zero rows. So, for $\lambda_{4}=\frac{n-1}{2}-\sqrt{(n-1) n+\left(\frac{n-1}{2}\right)^{2}}$, we have its algebraic multiplicity is 1 . We conclude that

$$
\operatorname{Spec}_{A}\left(\Gamma_{D_{2 n}}\right)=\left[\frac{n-1}{2}+\sqrt{(n-1) n+\left(\frac{n-1}{2}\right)^{2}} \quad \begin{array}{cccc}
0 & -1 & \frac{n-1}{2}-\sqrt{(n-1) n+\left(\frac{n-1}{2}\right)^{2}} \\
1 & n-2 & n-1
\end{array}\right]
$$

\section{Theorem 2:}

Let $\Gamma_{D_{2 n}}$ be a non commuting graph of dihedral group $D_{2 n}$ where $n$ is even positive integer and $n \geq 6$. The adjacency spectrum of $\Gamma_{D_{2 n}}$ is

$$
\operatorname{Spec}_{A}\left(\Gamma_{D_{2 n}}\right)=\left[\begin{array}{cccc}
\frac{n-2}{2}+\sqrt{\frac{5 n^{2}-12 n+4}{4}} & 0 & -2 & \frac{n-2}{2}-\sqrt{\frac{5 n^{2}-12 n+4}{4}} \\
1 & \frac{n-2}{2} & \frac{3 n-6}{2} & 1
\end{array}\right] .
$$

The next results are Laplacian spectrum of commuting and non commuting graph of dihedral group.

\section{Lemma 2:}

Let $C\left(D_{2 n}\right)$ be a commuting graph of dihedrral group $D_{2 n}$ where $n$ is positive integer and $n \geq 3$. The characteristic polynomial for Laplacian matrix $L\left(C\left(D_{2 n}\right)\right)$ of $C\left(D_{2 n}\right)$ is

and

$$
p(\lambda)=-\lambda(\lambda-n)^{n-2}(\lambda-1)^{n}(\lambda-2 n), \text { for odd } n
$$

$$
p(\lambda)=-\lambda(\lambda-n)^{n-3}((\lambda-4)(\lambda-2))^{\frac{n}{2}}(\lambda-2 n)^{2}, \text { for even } n
$$

\section{Theorem 3:}

Let $C\left(D_{2 n}\right)$ be a commuting graph of dihedral group $D_{2 n}$ where $n$ is odd positive integer and $n \geq 3$. The Laplacian spectrum of $C\left(D_{2 n}\right)$ is

Proof:

$$
\operatorname{Spec}_{L}\left(C\left(D_{2 n}\right)\right)=\left[\begin{array}{cccc}
2 n & n & 1 & 0 \\
1 & n-2 & n & 1
\end{array}\right] \text {. }
$$

From Lemma 2 , for odd $n$, we have $\lambda_{1}=2 n, \lambda_{2}=n, \lambda_{3}=1$, and $\lambda_{4}=0$ as eigenvalues for the Laplacian matrix of $C\left(D_{2 n}\right)$. And the multiplicity for each eigenvalue is $m\left(\lambda_{1}\right)=$ $1, m\left(\lambda_{2}\right)=n-2, m\left(\lambda_{3}\right)=n$, and $m\left(\lambda_{4}\right)=1$.

\section{Theorem 4:}

Let $C\left(D_{2 n}\right)$ be a commuting graph of dihedrral group $D_{2 n}$ where $n$ is even positive integer and $n \geq 6$. The Laplacian spectrum of $C\left(D_{2 n}\right)$ is

Proof:

$$
\operatorname{Spec}_{L}\left(C\left(D_{2 n}\right)\right)=\left[\begin{array}{ccccc}
2 n & n & 4 & 2 & 0 \\
2 & n-3 & \frac{n}{2} & \frac{n}{2} & 1
\end{array}\right]
$$

From Lemma 2, for even $n$ and $n \geq 6$, we have $\lambda_{1}=2 n, \lambda_{2}=n, \lambda_{3}=4, \lambda_{4}=2$, and $\lambda_{5}=0$ as eigenvalues for the Laplacian matrix of $C\left(D_{2 n}\right)$. And the multiplicity for each eigenvalue is $m\left(\lambda_{1}\right)=2, m\left(\lambda_{2}\right)=n-3, m\left(\lambda_{3}\right)=\frac{n}{2}, m\left(\lambda_{4}\right)=\frac{n}{2}$, and $m\left(\lambda_{5}\right)=1$.

From our investigation, the Laplacian spectrum of $C\left(D_{8}\right)$ is 
If we apply Theorem 4 for $n=4$ then we have

$$
\operatorname{Spec}_{L}\left(C\left(D_{8}\right)\right)=\left[\begin{array}{llll}
8 & 4 & 2 & 0 \\
2 & 3 & 2 & 1
\end{array}\right] .
$$

$$
\operatorname{Spec}_{L}\left(C\left(D_{8}\right)\right)=\left[\begin{array}{lllll}
8 & 4 & 4 & 2 & 0 \\
2 & 1 & 2 & 2 & 1
\end{array}\right]
$$

According to spectrum definition, we must write the Laplacian spectrum for $C\left(D_{8}\right)$ as

So, by this reason, Theorem 4 also holds for $n=4$.

$$
\operatorname{Spec}_{L}\left(C\left(D_{8}\right)\right)=\left[\begin{array}{llll}
8 & 4 & 2 & 0 \\
2 & 3 & 2 & 1
\end{array}\right]
$$

\section{Lemma 3:}

Let $\Gamma_{D_{2 n}}$ be a non commuting graph of dihedral group $D_{2 n}$ where $n$ is odd positive integer and $n \geq 3$. The characteristic polynomial for the Laplacian matrix $L\left(\Gamma_{D_{2 n}}\right)$ of $\Gamma_{D_{2 n}}$ is

$$
p(\lambda)=-\lambda(n-\lambda)^{n-2}((-2 n+1)+\lambda)^{n} .
$$

\section{Theorem 5:}

Let $\Gamma_{D_{2 n}}$ be a non commuting graph of dihedral group $D_{2 n}$ where $n$ is odd positive integer and $n \geq 3$. The Laplacian spectrum of $\Gamma_{D_{2 n}}$ is

Proof:

$$
\operatorname{Spec}_{L}\left(\Gamma_{D_{2 n}}\right)=\left[\begin{array}{ccc}
2 n-1 & n & 0 \\
n & n-2 & 1
\end{array}\right] \text {. }
$$

From Lemma 3, for odd $n$ and $n \geq 3$, we have $\lambda_{1}=2 n-1, \lambda_{2}=n$, and $\lambda_{3}=0$. as eigenvalues for the Laplacian matrix of $\Gamma_{D_{2 n}}$. And the multiplicity for each eigenvalue is $m\left(\lambda_{1}\right)=n, m\left(\lambda_{2}\right)=n-2$, and $m\left(\lambda_{3}\right)=1$

\section{Theorem 6:}

Let $\Gamma_{D_{2 n}}$ be a non commuting graph of dihedral group $D_{2 n}$ where $n$ is even positive integer and $n \geq 6$. The Laplacian spectrum of $\Gamma_{D_{2 n}}$ is

$$
\operatorname{Spec}_{L}\left(\Gamma_{D_{2 n}}\right)=\left[\begin{array}{cccc}
2(n-1) & 2(n-2) & n & 0 \\
\frac{n}{2} & \frac{n}{2} & n-3 & 1
\end{array}\right] .
$$

\section{Proof:}

The characteristic polynomial for the Laplacian matrix $L\left(\Gamma_{D_{2 n}}\right)$ of $\Gamma_{D_{2 n}}$ where $n$ is even positive integer and $n \geq 6$ is $p(\lambda)=-\lambda(n-\lambda)^{n-3}(2(n-1)+\lambda)^{\frac{n}{2}}(2(n-2)+\lambda)^{\frac{n}{2}}$

So we have $\lambda_{1}=2(n-1), \lambda_{2}=2(n-2), \lambda_{3}=n$, and $\lambda_{4}=0$ as eigenvalues for the Laplacian matrix of $\Gamma_{D_{2 n}}$. And the multiplicity for each eigenvalue is $m\left(\lambda_{1}\right)=\frac{n}{2}$, $m\left(\lambda_{2}\right)=\frac{n}{2}, m\left(\lambda_{3}\right)=n-3$, and $m\left(\lambda_{4}\right)=1$.

As for the adjacency spectrum, we just have the signless Laplacian spectrum for non commuting graph of dihedral group. We present the result without proof.

\section{Theorem 7:}

Let $\Gamma_{D_{2 n}}$ be a non commuting graph of dihedral group $D_{2 n}$ where $n$ is even positive integer and $n \geq 8$. The signless Laplacian spectrum of $\Gamma_{D_{2 n}}$ is

$$
\operatorname{spec}_{Q}\left(\Gamma_{D_{2 n}}\right)=\left[\begin{array}{ccccc}
(2 n-3)+\sqrt{2 n^{2}-8 n+9} & 2(n-2) & 2(n-3) & n & (2 n-3)-\sqrt{2 n^{2}-8 n+9} \\
1 & \frac{n}{2} & \frac{n-2}{2} & n-3 & 1
\end{array}\right]
$$


The last two theorems are results for detour spectrum of non commuting graph on dihedral group. We start by this lemma.

\section{Lemma 4:}

Let $\Gamma_{D_{2 n}}$ be a non commuting graph of dihedral group $D_{2 n}$ where $n$ is positive integer and $n \geq 3$. The longest path between two distinct vertices $x, y \in V\left(\Gamma_{D_{2 n}}\right)$ is $2 n-2$ for odd $n$ and $2 n-3$ for even $n$.

\section{Proof:}

For dihedral group $D_{2 n}=\left\{1, r, r^{2}, \ldots, r^{n-1}, s, s r, s r^{2}, \ldots, s r^{n-1}\right\}$ where $n$ is odd positive integer, $n \geq 3$, we have $Z\left(D_{2 n}\right)=\{1\}$. So, the non commuting graph $\Gamma_{D_{2 n}}$ of $D_{2 n}$ has $\{r$, $\left.r^{2}, \ldots, r^{n-1}, s, s r, s r^{2}, \ldots, s r^{n-1}\right\}$ as its vertex set. Hence the order of $\Gamma_{D_{2 n}}$ is $2 n-1$. Because $n$ is odd, we have $s r^{i} \neq r^{i} s, i=1,2, \ldots, n-1$, so $s$ and $r^{i}$ are adjacent in $\Gamma_{D_{2 n}}$. And also, $s r^{i} s r^{j} \neq s r^{j} s r^{i}$, for $i=1,2, \ldots, n-1$ and $j=1,2, \ldots, n-1$, where $i \neq j$, so $s r^{i}$ and $s r^{j}$ are adjacent in $\Gamma_{D_{2 n}}$. On the other hand $r^{i}$ and $r^{j}$ are not adjacent in $\Gamma_{D_{2 n}}$ because $r^{i} r^{j}=r^{j} r^{i}$ in $D_{2 n}$. We have that the sum of degree of any two non adjacent vertices in $\Gamma_{D_{2 n}}$ is $2 n>$ $\frac{2 n-1}{2}$ and $\Gamma_{D_{2 n}}$ is Hamiltonian graph (see [10]). So, the length of the longest path between two distinct vertices is $2 n-2$ for odd $n$.

For dihedral group $D_{2 n}=\left\{1, r, r^{2}, \ldots, r^{n-1}, s, s r, s r^{2}, \ldots, s r^{n-1}\right\}$ where $n$ is even positive integer, $n>3$, we have $Z\left(D_{2 n}\right)=\left\{1, r^{\frac{n}{2}}\right\}$. So, the non commuting graph $\Gamma_{D_{2 n}}$ of $D_{2 n}$ has $\left\{r, r^{2}, \ldots, r^{\frac{n}{2}-1}, r^{\frac{n}{2}+1}, \ldots, r^{n-1}, s, s r, s r^{2}, \ldots, s r^{n-1}\right\}$ as its vertex set. Hence the order of $\Gamma_{D_{2 n}}$ is $2 n-2$. We also have that $\Gamma_{D_{2 n}}$ is Hamiltonian graph. So, the length of the longest path between two distinct vertices is $2 n-3$ for even $n$.

\section{Theorem 8:}

Let $\Gamma_{D_{2 n}}$ be a non commuting graph of dihedral group $D_{2 n}$ where $n$ is odd positive integer and $n \geq 3$. The detour spectrum of $\Gamma_{D_{2 n}}$ is

$$
\operatorname{Spec}_{D D}\left(\Gamma_{D_{2 n}}\right)=\left[\begin{array}{cc}
(2 n-2)^{2} & -(2 n-2) \\
1 & 2 n-2
\end{array}\right] .
$$

\section{Proof:}

By Lemma 4 , we have $D D\left(\Gamma_{D_{2 n}}\right)$ is $(2 n-1) \times(2 n-1)$ matrix where all entries in its main diagonal is 0 and $2 n-2$ elsewhere, for odd $n$. Then, we have the characteristic polinomial is $p(\lambda)=(\lambda+(2 n-2))^{2 n-2}\left(\lambda-(2 n-2)^{2}\right)$. From the characteristics polinomial, we have $\lambda_{1}=(2 n-2)^{2}$ and $\lambda_{2}=2 n-2$ as eigenvalues of $D D\left(\Gamma_{D_{2 n}}\right)$ with their multiplicity are $m\left(\lambda_{1}\right)=1$ and $m\left(\lambda_{2}\right)=2 n-2$. So, we get the desired result.

\section{Theorem 9:}

Let $\Gamma_{D_{2 n}}$ be a non commuting graph of dihedral group $D_{2 n}$ where $n$ is odd positive integer and $n \geq 3$. The detour spectrum of $\Gamma_{D_{2 n}}$ is

\section{Proof:}

$$
\operatorname{SpecDD}\left(\Gamma_{D_{2 n}}\right)=\left[\begin{array}{cc}
(2 n-3)^{2} & -(2 n-3) \\
1 & 2 n-3
\end{array}\right] .
$$

By Lemma 4, we have $D D\left(\Gamma_{D_{2 n}}\right)$ is $(2 n-2) \times(2 n-2)$ matrix where all entries in its main diagonal is 0 and $2 n-3$ elsewhere, for even $n$. Then, we have the characteristic polinomial is $p(\lambda)=(\lambda+(2 n-2))^{2 n-2}\left(\lambda-(2 n-2)^{2}\right)$. From the characteristic polinomial, we have $\lambda_{1}=(2 n-3)^{2}$ and $\lambda_{2}=2 n-3$ as eigenvalues of $D D\left(\Gamma_{D_{2 n}}\right)$ with their multiplicity are $m\left(\lambda_{1}\right)=1$ and $m\left(\lambda_{2}\right)=2 n-3$. This lead us to the detour spectrum. 


\section{CONCLUSION}

From our results, several spectrums for several cases of $n$ are not determined yet. So, the remaining cases can be done in further research. Investigation for spectra of commuting and non commuting graph of symmetric group are also interesting to be done.

\section{REFERENCES}

[1] Abdussakir, N. N. Azizah, and F. F. Novandika, Teori Graf. Malang: UIN Malang Press, 2009.

[2] G. Chartrand, L. Lesniak, and P. Zhang, Graphs and digraphs, 6th Edition. Florida: Chapman and Hall, 2015.

[3] B. Mohar, "Laplace eigenvalues of graphs-a survey," Discrete Math., vol. 109, no. 1-3, pp. 171-183, 1992.

[4] A. E. Brouwer and W. H. Haemers, Spectra of graphs: Monograph. New York: Springer, 2011.

[5] S. K. Ayyaswamy and S. Balachandran, "On detour spectra of some graphs," Int. J. Math. Comput. Phys. Electr. Comput. Eng., vol. 4, no. 7, pp. 1038-1040, 2010.

[6] N. Biggs, Algebraic graph theory, 2nd Edition. New York: Cambridge University Press, 1993.

[7] S. Yin, "Investigation on spectrum of the adjacency matrix and Laplacian matrix of graph G1,” WSEAS Trans. Syst., vol. 7, no. 4, pp. 362-372, 2008.

[8] A. Nawawi and P. Rowley, "On commuting graphs for elements of order 3 in symmetric groups," Electron. J. Comb., vol. 22, no. 1, pp. 1-21, 2015.

[9] A. Abdollahi, A. Azad, A. M. Hassanabadi, and M. Zarrin, "On the clique numbers of non-commuting graphs of certain groups," Algebr. Colloq., vol. 17, no. 4, pp. 611-620, 2010.

[10] A. Abdollahi, S. Akbari, and H. R. Maimani, "Non-commuting graph of a group," $J$. Algebr., vol. 298, no. 2, pp. 468-492, 2006.

[11] S. R. Jog and R. Kotambari, "On the adjacency, Laplacian, and signless Laplacian spectrum of coalescence of complete graphs," J. Math., vol. 2016, pp. 1-11, 2016.

[12] S. Akbari, M. Ghandehari, M. Hadian, and A. Mohammadian, "On commuting graphs of semisimple rings," Linear Algebra Appl., vol. 390, no. 1-3, pp. 345-355, 2004.

[13] M. R. Darafsheh, "Groups with the same non-commuting graph," Discret. Appl. Math., vol. 157 , no. 4, pp. 833-837, 2009.

[14] J. Vahidi and A. A. Talebi, "The commuting graphs on groups D2n and Qn," J. Math. Comput. Sci., vol. 1, no. 2, pp. 123-127, 2010.

[15] H. Rahayuningtyas, Abdussakir, and A. Nashichuddin, "Bilangan Kromatik Graf Commuting dan Non Commuting Grup Dihedral," CAUCHY, vol. 4, no. 1, pp. 16-21, 2015.

[16] T. T. Chelvam, K. Selvakumar, and S. Raja, "Commuting graphs on dihedral group main results," J. Math. Comput. Sci., vol. 2, no. 2, pp. 402-406, 2011.

[17] T. Woodcock, "The commuting graph of the symmetric group Sn," Int. J. Contemp. Math. Sci., vol. 10, no. 6, pp. 287-309, 2015. 